\title{
In-Situ Plasmonic Tracking Oxygen Evolution Revealing Multistage Oxygen Diffusion and Accumulating Inhibition
}

\section{Jungang Wang}

School of Chemistry and Molecular Engineering, East China University of Science and Technology

\section{Lifang Shi}

Institute of Optics and Electronics, Chinese Academy of Sciences,

\section{Yingying Su}

East China Normal University

\section{Liwei Liu}

Institute of Optics and Electronics, Chinese Academy of Sciences,

\section{Jin Xie}

Institute of Microelectronics, Chinese Academy of Sciences

\section{Yang Tian}

East China Normal University

Di Li ( $\nabla$ dli@chem.ecnu.edu.cn )

East China Normal University https://orcid.org/0000-0003-1674-0110

\section{Article}

Keywords: Mass Transfer Processes, Gas Evolution Reactions, Electrode-electrolyte Interface, Nanostructured Plasmonic Interface, Programmable Potential Scan Strategy

Posted Date: November 19th, 2020

DOl: https://doi.org/10.21203/rs.3.rs-104930/v1

License: (a) (1) This work is licensed under a Creative Commons Attribution 4.0 International License. Read Full License

Version of Record: A version of this preprint was published at Nature Communications on April 12th, 2021. See the published version at https://doi.org/10.1038/s41467-021-22434-3. 
In-Situ Plasmonic Tracking Oxygen Evolution Revealing Multistage Oxygen Diffusion and Accumulating Inhibition

Jun-Gang Wang, ${ }^{1}$ Lifang Shi, ${ }^{3}$ Yingying Su,,${ }^{1}$ Liwei Liu, ${ }^{3}$ Jing Xie, ${ }^{4}$ Yang Tian, ${ }^{1}$ and Di Li* ${ }^{1,2}$

${ }^{1}$ School of Chemistry and Molecular Engineering, East China Normal University, Shanghai 200241, China

${ }^{2}$ Key Laboratory of Bioorganic Phosphorus Chemistry \& Chemical Biology, Department of Chemistry, Tsinghua University, Beijing, 10084, China

${ }^{3}$ Institute of Optics and Electronics, Chinese Academy of Sciences, Chengdu 610209, China

${ }^{4}$ Institute of Microelectronics, Chinese Academy of Sciences, Beijing 100029, China 


\begin{abstract}
Understanding mass transfer processes concomitant with electrochemical conversion for gas evolution reactions at the electrode-electrolyte interface plays a key role in advancing renewable energy storage and conversion. However, due to the complicated diffusion behavior of gas at the dynamic catalytic interfaces, it is still a great challenge to accurately portray mass transfer of gas during electrocatalysis. Here, we track the diffusion of dissolved oxygen on $\mathrm{Cu}$ nanostructured plasmonic interface, reveal multistage oxygen diffusion behaviors, including premature oxygen accumulation, spontaneous diffusion and accelerated oxygen dissipation, and indicate an accumulating inhibition effect on oxygen evolution arising from interfacial dissolved oxygen. With these knowledges, we develop a programmable potential scan strategy to eliminate interfacial gas products that alleviates the concentration polarization, releases accessible actives sites and promotes electrocatalytic performance. Our findings provide a direct observation of the interfacial mass transfer processes that govern the multi-phases catalysis kinetics.
\end{abstract}




\section{Introduction}

Electrode-electrolyte interfaces (EEIs), where mass transfer and energy transfer proceeds concomitantly, are of paramount significance in electrochemistry. ${ }^{1-3}$ Oxygen evolution reaction (OER), plays a pivotal role in water splitting by providing protons and electrons at the EEIs, however its sluggish kinetics limits their performance and commercialization. ${ }^{4-6}$ Gas evolution leads to an undesirable blockage of redox reaction sites and ion conducting pathways, resulting in an increase of ohmic resistance and formation of a heterogeneous concentration gradient at the EEIs. All these effects give rise to energy losses and unfavorable attenuation of electrochemical conversion efficiencies. Moreover, under different oxygen concentration gradients, the electrode interfaces experience different oxygen compositions $\left(\mathrm{OH}^{-}, \mathrm{OH}^{*}\right.$, dissolved oxygen and oxygen bubble, etc.) at various polarization. This, in turn, generates varying operation regimes in terms of either mass transport or kinetic reaction limitations for the electrocatalytic processes, leading to confusing of interfacial reaction mechanism. ${ }^{7}$ The dynamics of oxygen concentration gradients at the EEIs thus remains quite elusive since it is readily hidden by vast spectators and electrolytes, and is often overlooked during kinetic analysis. Therefore, it is of crucial to precisely identify various oxygen diffusion stages under operando conditions and uncover the influence of oxygen concentration gradients on OER performance. ${ }^{8}$

Conventional electrochemical measurements, however, are insufficient to identify dissolved oxygen and uncover the dynamic dissolved oxygen diffusion processes, because the limitation of direct molecular recognition of interfacial dissolved oxygen and the complexity induced by non-Faradaic processes, such as concomitant mass transfer of spectator species and multi-phase 
transition. In-situ spectroelectrochemical techniques ${ }^{9-11}$, such as X-ray absorption/diffraction/scattering spectroscopy ${ }^{12,13}$, infrared and Raman spectroscopy ${ }^{14}$, mass spectrometry $^{15,16}$ and nuclear magnetic resonance ${ }^{17}$ are powerful tools for identifying real accessible catalytic active sites, adsorbed intermediates, and monitoring the reconstruction of electrocatalysts under operando conditions. Unfortunately, these approaches also lack the ability of identification fluctuations of interfacial dissolved oxygen due to the inherent negligible weak interaction between dissolved oxygen and the electrode surface. Thus, it is still a great challenge for current techniques to in-situ observe oxygen evolution on heterogeneous catalytic interfaces and distinguish the diffusion profile of dissolved oxygen within the interfacial electric field.

Plasmonic-enhanced spectroscopy ${ }^{18,19}$ exploits the excellent optical performance of advanced plasmonic nanostructures and possesses noninvasive feature with superior sensitivity and compatibility in monitoring a variety of chemical processes, including heterogeneous catalysis $^{20-22}$, electrochemistry ${ }^{23-25}$ and phase transition ${ }^{26-28}$. Thereby, plasmonic-enhanced spectroscopy may possess the ability to differentiate oxygen compositions during OER at EEIs.

In the present study, we establish a dual-functional plasmonic $\mathrm{Cu}$ nanoparticles (NPs) interface that amenable to host electrochemical reaction and synchronous plasmonic extinction dissection. Harnessing the environment refractive sensitive plasmonic nanostructures, in-situ extinction spectroscopy enables identification of oxygen composition changes during OER by analyzing small spectral variations induced by the dynamics of interfacial dissolved oxygen profile at the EEIs. ${ }^{29,30}$ By comparing synchronized plasmonic and electrochemical responses, we uncover not only a multistage of oxygen diffusion at the EEIs, but also an accumulating 
inhibition effect from confined mass transfer of oxygen on OER. The identification of multistage of oxygen diffusion and accumulating inhibition effect suggests that removing the freshly evolved $\mathrm{O}_{2}$ from active sites may not only accelerate the charge transfer process and but also recover catalytic sites, leading to improved OER performance. We thereby develop a programmable potential scan strategy to electrochemically reduce freshly generated $\mathrm{O}_{2}$ to prevent its accumulating at the EEIs. The proposed programmable potential scan strategy results in significantly improved OER performance, which shows a potential to be a general operation in OER experiments. With the ability to deliver critical interfacial reaction information, the proposed strategy will provide in-depth information for understanding the intrinsic potential-dependent dynamic processes of various gas involving systems at the EEIs.

\section{Results}

Seed-Mediated Electrodeposition of Plasmonic Cu NPs. Conventional electrochemical measurements are incapable of in-situ probing the oxygen diffusion behavior because of (i) severe polarization effect induced by the increased chemical potential of dissolved gas molecules at the electrode interface; (ii) perturbations from non-Faradaic portion and concomitant side interfacial reactions at the electrode surface-liquid/gas diffusion layer, thereby unable to reveal the dynamic oxygen evolution at the EEIs. By taking advantage of the near-plasmonic enhancement, plasmonic-enhanced extinction spectroscopy provides an alternative way for real-time monitoring OER and revealing oxygen concentration profiles under polarization.

Here, we developed a plasmonic $\mathrm{Cu}$ NPs electrode as a platform to explore OER. This 

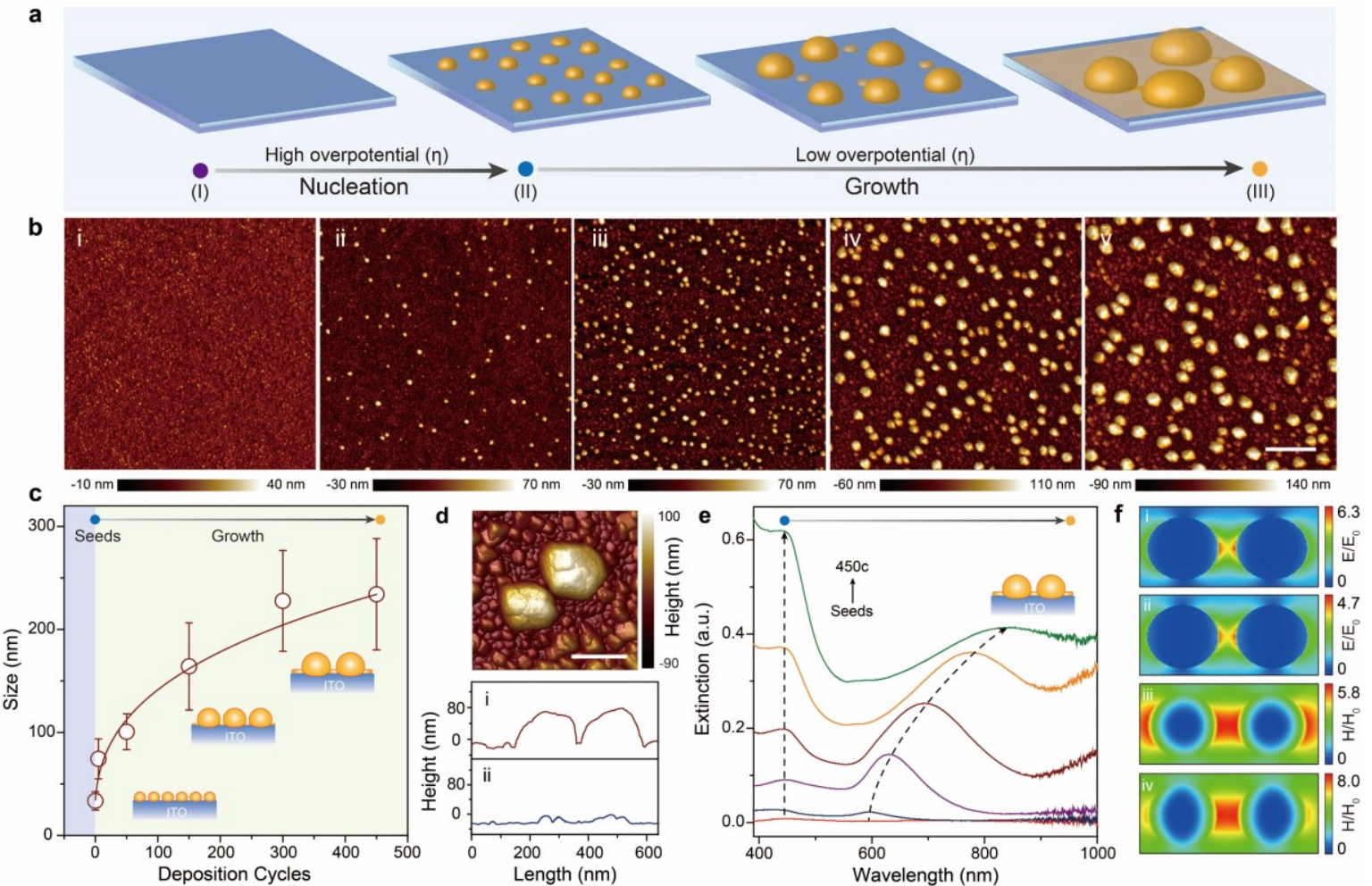

Fig. 1. Seed-mediated electrodeposition of plasmonic $\mathrm{Cu}$ nanoparticles. (a) Schematic illustrating the electrochemical seed-mediated growth strategy. (b) AFM images of $\mathrm{Cu}$ seeds (i) and $\mathrm{Cu}$ NPs at different deposition cycles (ii 5, iii 50, iv 150 and v 300 cycles) (scale bar: $1 \mu \mathrm{m}$ ). (c) The correlation between $\mathrm{Cu}$ NPs size and deposition cycles. (d) High-resolution AFM image of Cu NPs (top, 150 cycles, scale bar: $200 \mathrm{~nm}$ ) and linear scan across $\mathrm{Cu}$ NPs dimmer (i) and nearby regions (ii) (bottom). (e) Ex-situ extinction spectra of Cu seeds and Cu NPs at different deposition cycles (5, 50, 150, 300, 450 cycles). (f) Top and side-view of electric (i and ii) and magnetic (iii and iv) field snapshots for the near field confinement at the plasmonic resonance wavelengths (i, iii $450 \mathrm{~nm}$ and ii, iv $690 \mathrm{~nm})$.

platform was served as both electrocatalytic unit and spectroscopically addressable component to host multiple in-situ spectroelectrochemical techniques. Briefly, $\mathrm{Cu}$ NPs was grown on conductive ITO substrates through a seed-mediated electrodeposition (Fig. 1a, detailed discussions see Supplementary Fig. 1-5). AFM images of Cu NPs at different deposition cycles (Fig. 1b) indicated $\mathrm{Cu}$ nuclei (ca. $34 \mathrm{~nm}$ in diameter) was appeared and homogeneously distributed on ITO after initial seeding process (Fig. 1b, i). An instantaneous nucleation was suggested as dominating process instead of progressive nucleation. ${ }^{31}$ With the increase of electrodeposition cycles, possible fusions of $\mathrm{Cu}$ nuclei in the proximity occurred, resulting in an enlargement of particle size, $\mathrm{Cu}$ NPs grew from $34 \mathrm{~nm}$ seeds at 0 cycle to maximum 234 
$\mathrm{nm}$ at 450 cycles (Fig. 1b-c and Supplementary Fig. 6, detailed composition analysis see Supplementary, Fig. 7). In addition, a particle stacked film was formed because of high $\mathrm{Cu}$ nuclei coverage, as confirmed by the high-resolution AFM image (Fig. 1d).

We then examined the extinction spectra of $\mathrm{Cu}$ NPs electrodes as a function of deposition cycles to reveal the correlation between $\mathrm{Cu}$ NPs structure and its optical response (Fig. 1e). Once a conductive film interface is formed beneath $\mathrm{Cu}$ nanoparticles, its plasmonic wave function involves a superposition of a charge transferplasmon (CTP) mode in monopolar modes of individual nanoparticle. As the reduction of the separation distance between adjacent nanoparticles and junction resistance, CTP experiences a remarkable monotonic red-shift. ${ }^{32,33}$ Therefore, the noticeable red-shift of plasmonic resonance band in Fig. 1d was assigned to the conductive connection between $\mathrm{Cu}$ NPs and the underlying film. Meanwhile, another plasmonic resonance mode at around $450 \mathrm{~nm}$ remained stable whereas its intensity increased with the electrodeposition cycles, which was attributed to the Ohmic dissipation associated with the charge transport across the junction and the reduction of the capacitive coupling. ${ }^{34}$

Furthermore, finite-difference time-domain (FDTD) simulations provide more details about the plasmonic response of the NP-on-conductive film stacked structure. A strong coupling between the adjacent $\mathrm{Cu}$ NPs excited by a plane wave and polarized toward the interparticle axis gave rise to a low energy, charge transfer plasmon resonance band at $690 \mathrm{~nm}$, and a high energy, screened bonding plasmonic resonance band at $450 \mathrm{~nm}$ (Fig. 1f), promoted by the conduction through the substrate. For both plasmonic modes, the induced electric and magnetic fields were confined in the vicinity of the gap region. ${ }^{35,36}$ The experimentally recorded extinction spectra revealed a good agreement with the simulated extinction spectra 
(Supplementary Fig. 8). The energy of CTP mode is known to be sensitive to the change of environmental refractive index, therefore, it is essential to convert subtle environmental change in gas evolution reaction into dynamic changes of extinction spectra.

\section{Dissolved Oxygen Diffusion Profile Monitored by a Single-Wavelength Plasmonic}

Strategy. The beneficial interplay between large electrochemical surface area and interfacial high active species contributed to the high catalysis performance of copper materials (detailed electrochemical measurements and discussion of oxygen evolution see Supplementary Fig. 910). The plasmonic $\mathrm{Cu}$ NPs electrode was then used to optically exploit oxygen diffusion behavior at the EEIs by taking advantage of the refractive sensitive plasmonic structure (Fig. 2a). Electrochemical steady-state extinction spectroscopy was employed to monitor OER (Fig. $2 b)$. Under enhanced anodic polarization from -1.10 to $-0.30 \mathrm{~V}(v s$. Pt, all the potentials were referenced to a Pt quasi-reference electrode unless otherwise stated), we observed a remarkable increase of extinction peak intensity ( $\mathrm{ca} .15 \%$ ) and a $27 \mathrm{~nm}$ peak red-shift (from 623 to 650 $\mathrm{nm}$ ), attributing to the electrochemical formed $\mathrm{Cu}$ oxides and hydroxides (Supplementary Fig. 9). Notably, the extinction intensity at $623 \mathrm{~nm}$ was increased $c a .14 .9 \%$ at $-0.30 \mathrm{~V}$ compared to that at $-1.10 \mathrm{~V}$. The extinction peak intensity was decreased $c a .70 .5 \%$ and shifted to 720 $\mathrm{nm}$ from $-0.30 \mathrm{~V}$ to $0.90 \mathrm{~V}$. These indicate that the $\mathrm{Cu}$ oxide and hydroxides intermediates were weakly bound and readily dissolved during long time anodic polarization, which hampered interfacial processes dissection under steady-state conditions.

We then developed a single-wavelength plasmonic monitoring strategy to precisely identify the diffusion behavior of dissolved oxygen (Fig. 2c). Generally, the interfacial processes at the EEIs could induce a fluctuation of nearby refractive index, leading to a systematic transition 

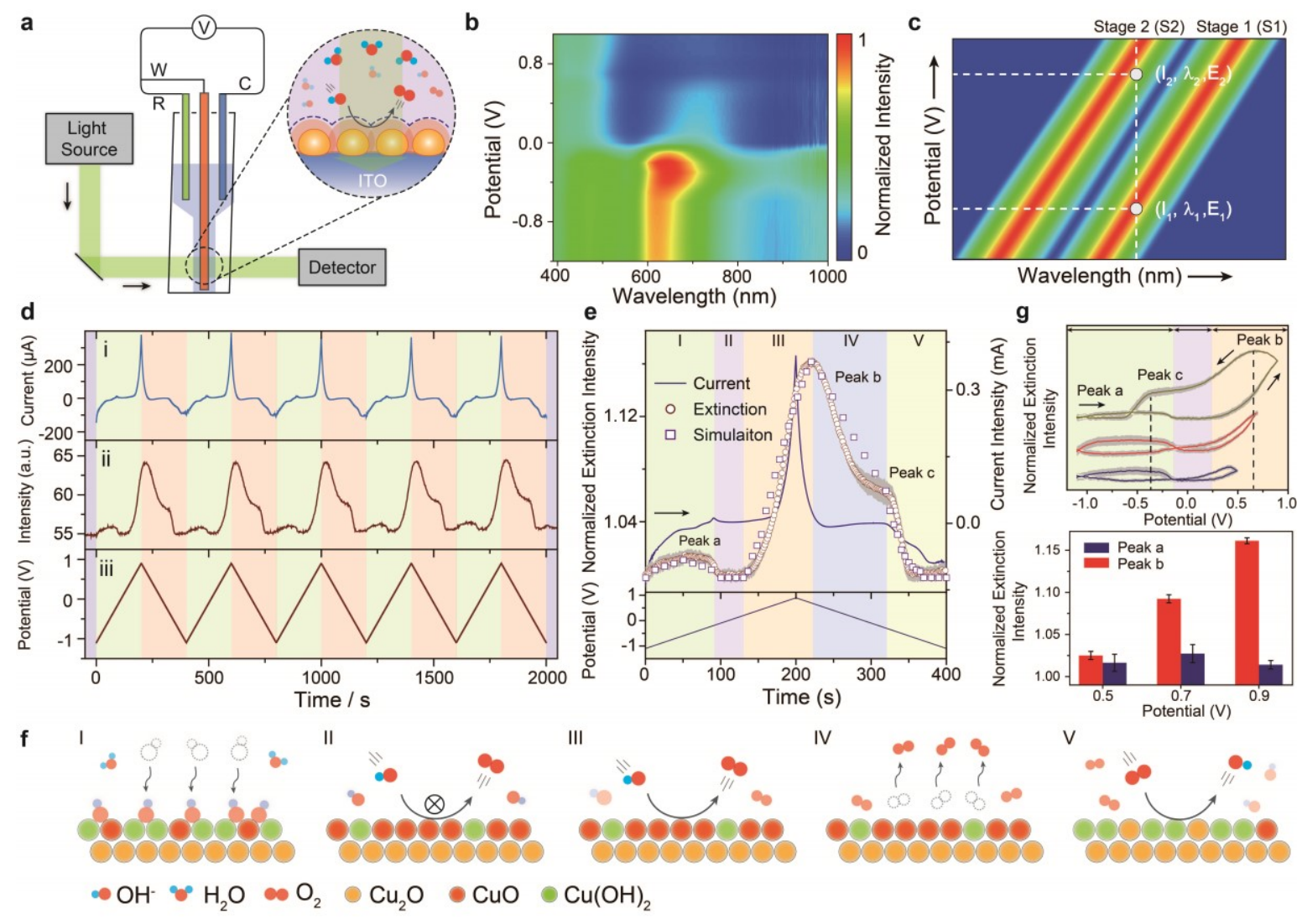

IV

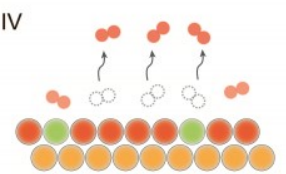

$\checkmark$

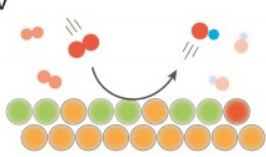

Fig. 2 Plasmonic monitoring oxygen evolution reaction. (a) Experimental setup of in-situ electrochemical extinction microscopy. (b) The normalized dynamic extinction intensity (contour plots) of a Cu NPs electrode under steady-state anodic polarization. (c) Schematic illustration of the plasmonic single-wavelength strategy for real-time monitoring OER at the EEIs. (d) The consecutive electrochemical current (i) during OER and synchronous extinction signal (ii) under dynamic potential scan (iii). (e) The representative dynamic extinction trajectory (o), current curve (-) and FDTD simulation ( $\square$ ) for OER on a plasmonic Cu NPs electrode scanned from -1.10 to $0.90 \mathrm{~V}$ at a scan rate $0.01 \mathrm{~V} \mathrm{~s}^{-1}$. (f) Schematic illustration of the interfacial reaction mechanism during dynamic potential scan. (g) The extinction voltammetry with different terminational potential $(0.50,0.70$, $0.90 \mathrm{~V}$ ) at a scan rate of $0.01 \mathrm{~V} \mathrm{~s}^{-1}$ (top panel). The dark region represents the standard error during consecutive five scan. The potential dependent of extinction intensity for peak $a$ and $b$ (bottom panel).

from original state $\left(\mathrm{S}_{1}, I_{1}-\lambda_{1}-E_{1}\right)$ to reaction state $\left(\mathrm{S}_{2}, I_{2}-\lambda_{2}-E_{2}\right)$. Therefore, the fluctuation of the extinction intensity at a constant $\lambda$ during dynamic potential scan, provides an ultrasensitive feedback of the interfacial reaction information at the EEIs (detailed discussions of this concept see Supporting Information Section 5). Of note, the NP-on-conductive film structure benefits the super sensitivity of the CTP mode $\left(\lambda_{\mathrm{CTP}}\right)$, which enables a precise recognition of delicate interfacial processes at the EEIs. Series control experiments with $\mathrm{Cu}$ NPs electrode performed under various potential scan range and scan rate revealed negligible extinction fluctuations 
(Supplementary Fig. 11-18). Despite weak anodic polarization current peak assigned to oxygen evolution was identified on bare ITO electrodes, the temporal optical readout cannot be readily observed in the absence of plasmonic-enhancement elements.

Accordingly, cyclic voltammetry and in-situ extinction voltammetry measurements were performed synchronously on a homemade three-electrode micro-electrochemical cell to explore oxygen evolution profile during OER. The reversible fluctuation of extinction intensity was in good agreement with current response (Fig. 2d). These periodic behaviors were attributed to the dynamic structural changes of the EEIs that involves both electrochemical redox of $\mathrm{Cu}$ NPs and oxygen evolution. Comparing this temporal extinction trajectory with current response, five typical potential regions were identified to explain the dynamic interfacial processes (Fig. 2e and Supplementary Fig. 9). These regions were: (I) active region, where the oxidation of $\mathrm{Cu}$ occurs; (II) passive region, where oxygen evolution is suppressed; (III) transpassive region, where the onset oxygen evolution region is controlled by reactive kinetics; (IV) diffusion region, where oxygen diffusion regions is dominated by diffusion kinetics and (V) reductive region, where oxygen reduction occurs (Fig. 2e, f). In particular, in Region I while the potential was scanned from -1.10 to $-0.36 \mathrm{~V}$, the extinction intensity was gradually increased under enhanced anodic polarization, indicating the phase growth associated within the oxide layer, ascribed to a temporal evolution of $\mathrm{Cu}_{2} \mathrm{O}$ and $\mathrm{CuO} / \mathrm{Cu}(\mathrm{OH})_{2}$ phases. In contrast, a rapid attenuation of the extinction intensity was observed after $-0.36 \mathrm{~V}$. These observations implied that potential-driven composition evolution of $\mathrm{Cu}$ oxides and hydroxides provide abundant vacancy sites for surface adsorption of $\mathrm{OH}$ to facilitate oxygen evolution through diminish the $\mathrm{OH} / \mathrm{O}$ ratio by releasing $\mathrm{H}$ to form desirable crystallization. ${ }^{37}$, 
${ }^{38}$ During the passive regions $(>-0.10 \mathrm{~V})$, indistinctive extinction fluctuation indicated that the ratio of oxide to hydroxide species remained stable in the oxide layer (II).

Interestingly, when the potential was swept to transpassive regions $(>0.20 \mathrm{~V}$, III), the transformation from an amorphous oxide of $\mathrm{CuO} / \mathrm{Cu}(\mathrm{OH})_{2}$ phases to a crystalline structure generated sufficient electron-conductive paths through the anodic oxide film for oxygen evolution. Therefore, the rapid increase in extinction intensity could be reasonably attributed to the oxygen evolution. ${ }^{39}$ Notably, when comparing extinction voltammetry with the linear potential scan from 0 to $0.90 \mathrm{~V}$, a significant lower onset potential for oxygen evolution was observed in extinction measurements $(c a .0 .40 \mathrm{~V})$. To understand the origin of this distinction in onset potential, both current and extinction voltammetry was fitted based on a onedimensional diffusion model (see derivation in Supplementary Fig. 19 and 20).

$$
\eta_{c}=\frac{R T}{n F} \ln \left(\Phi\left(O H^{-}\right)\left(1-\frac{i}{i_{l, O H^{-}}}\right)\right)^{4}\left(\Phi\left(O_{2}\right)\left(1-\frac{i}{i_{l, O_{2}}}\right)\right)^{-1}
$$

where $T$ is the temperature, $R$ is the gas constant, $n$ is the electron transfer number and $F$ is the Faraday's constant, $i_{1}$, OH- and $i_{1}, \mathrm{O} 2$ are the limiting current density of $\mathrm{OH}^{-}$oxidation (anodic polarization) and $\mathrm{O}_{2}$ reduction (cathodic polarization), respectively. The partition coefficient $\Phi$ indicates the solubility of a species in the diffusion layer and can be defined as the ratio of the equilibrium concentration in the diffusion layer to the value in the bulk electrolyte. ${ }^{40}$

From the fitting line, the concentration overpotential incurred by the oxygen diffusion layer was estimated to be $c a$. $0.20 \mathrm{~V}$. Under enhanced oxygen concentration polarization, the measured current signals comprised not only the Faradaic components but also the nonFaradaic portion, both contributing to the remarkable distinction in the onset potential. Since the extinction intensity is directly correlated with the amounts of interfacial dissolved oxygen, 
it represents only the absolute Faradaic current, resulting diminishing interference from nonFaradaic contribution, therefore enables precise identification of intrinsic onset potential at an early stage of the OER. Subsequently, the extinction intensity showed a gradually attenuation with decreasing of potential $(0.67 \mathrm{~V}$ to $-0.63 \mathrm{~V})$, indicating the diffusion of dissolved oxygen far away from the EEIs. It was worth noting that the diffusion of dissolved oxygen followed two different modes (Fig. 2e, IV and V), a spontaneous convection with a decay rate of $1.0 \times 10^{-}$ ${ }^{3} \mathrm{~s}^{-1}(0.67 \mathrm{~V}$ to $-0.35 \mathrm{~V})$ and an accelerated diffusion with a higher decay rate of $2.5 \times 10^{-2} \mathrm{~s}^{-1}$ $(-0.35 \mathrm{~V}$ to $-0.63 \mathrm{~V})$. Due to the enhanced cathodic polarization, the newly formed interfacial species $\left(\mathrm{Cu}_{2} \mathrm{O} / \mathrm{Cu}(\mathrm{OH})_{2}\right)$ and near negative electric field diminishes the desorption of oxygen and accelerates the dissipation of dissolved oxygen near the electrode surface.

We then established an interfacial reaction model through simulations of adsorbed chemical species and potential- dependent gas evolution at the EEIs to illustrate how the dynamic interfacial composition affects the plasmonic extinction spectro scopy (Fig. 2e). As the enhancement of anodic polarization, the phase transition from $\mathrm{Cu}_{2} \mathrm{O}$ to $\mathrm{CuO}$ and $\mathrm{Cu}(\mathrm{OH})_{2}$ phases led to the reduction of the refractive index from 2.90 to 2.72 and 1.71 , resulting an increasing of the extinction intensity. Further deprotonation in $\mathrm{CuO} / \mathrm{Cu}(\mathrm{OH})_{2}$ phases caused the growth of refractive index from 1.71 to 2.72 , that accounts for the gradual attenuation in extinction intensity. When the oxygen evolution occurred, the dissolved oxygen accumulated at the electrode surface caused the decrease of local electrolyte refractive index, evidenced by the promptly increased spectroscopic intensity (Supplementary Fig. 21). More importantly, the spontaneous convection and accelerated diffusion of oxygen on the electrode surface during cathodic polarization resulted in the recovery of local interfacial environment and the 

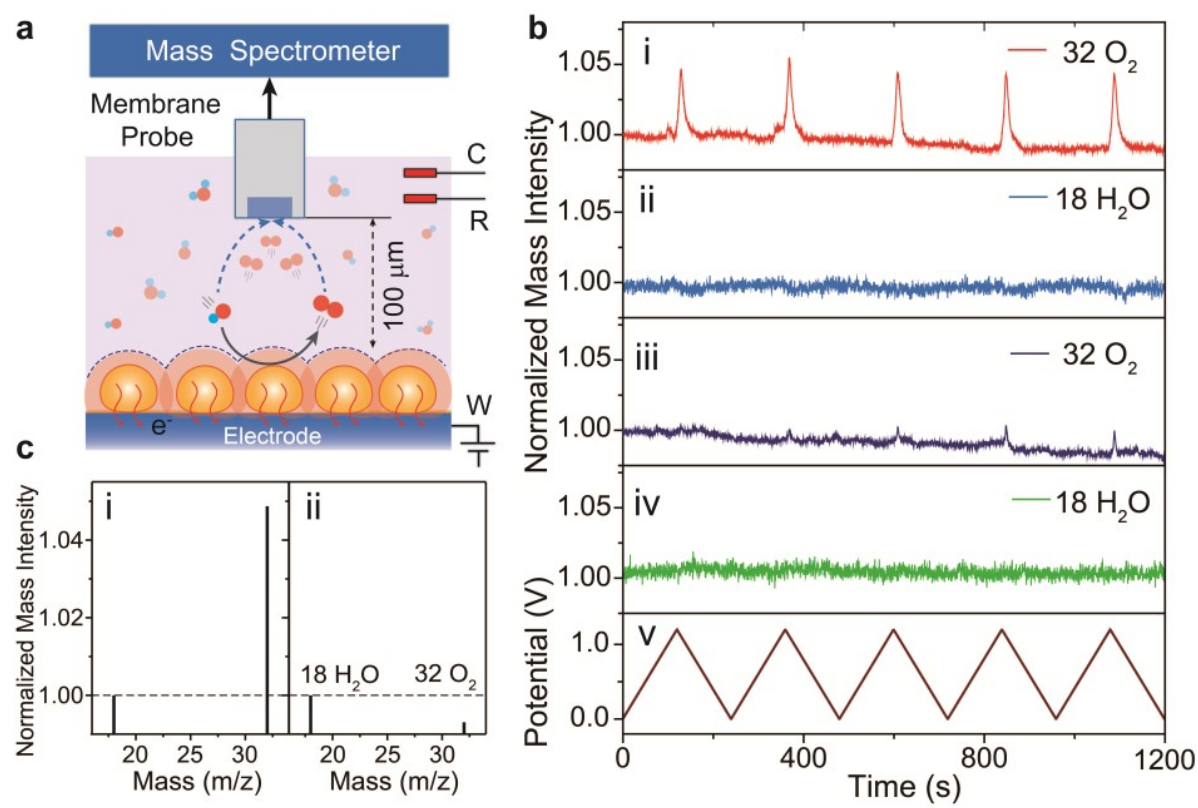

Fig. 3 Potential dependent mass spectrometry of dissolved oxygen. (a) Schematic of in-situ differential electrochemical mass spectrometry. (b) Dynamic mass intensity of $\mathrm{O}_{2}$ and $\mathrm{H}_{2} \mathrm{O}$ analyzed by in-situ DEMS during cyclic voltammetry in the potential range from 0 to $1.10 \mathrm{~V}$ on working electrode $\mathrm{Cu} / \mathrm{glass}$ carbon-disc (i, ii) and glass carbon-disc (iii) and (iv), respectively, in a homemade micro-electrochemical cell in $0.1 \mathrm{M} \mathrm{KOH}$ solution, at a scan rate of $0.01 \mathrm{~V} \mathrm{~s}^{-1}$. (c) The mass spectra during dynamic potential scan on the working electrode $\mathrm{Cu} / \mathrm{glass}$ carbon-disc (i) and glass carbon-disc (ii).

corresponding simulated spectroscopic trends were in good accordance with experimental results, indicating that the proposed interfacial reaction model captured the intrinsic features of the oxygen evolution reaction on the plasmonic electrode interface. These results explicitly illustrated the dynamic oxidation of $\mathrm{Cu}$ plasmonic surface and uncovered the presence of multistage diffusion of dissolved oxygen at the EEIs. Additionally, the extinction peak $b$ appeared at $0.67 \mathrm{~V}$ was assigned to the oxygen evolution at the EEIs, and the intensity of this peak increased quickly with enhancement of anodic polarization $(>0.50 \mathrm{~V}$, Fig. $2 \mathrm{~g})$. Meanwhile, the extinction peak a that assigned to the electrogenerated $\mathrm{Cu}$ oxides and hydroxides showed an obsolete potential-dependent relationship and remained stable in intensity with increasing anodic polarization, indicating an evident accumulation of dissolved oxygen at the EEIs under OER condition.

We further sought Mass spectrometer to provide direct molecular evidence to solidify that 
dynamic extinction spectra variations were results of electrochemical-generated oxygen rather than the other gas, for example, air. Mass spectrometer coupled with electrospray ionization was adopted to in-situ analyze oxygen and water (Fig. 3). The oxygen $\left(\mathrm{O}_{2}, 32 \mathrm{~m} / \mathrm{z}\right)$ was captured in the negative mode with predominant intensity than the internal reference mass intensity $\left(\mathrm{H}_{2} \mathrm{O}\right.$, $18 \mathrm{~m} / \mathrm{z}$ ). No other mass signals were observed. Notably, the remarkable periodic enhancement ca. 7 times of the oxygen mass intensity than that on bare glass carbon electrode during consecutive anodic polarization from 0 to $1.10 \mathrm{~V}$, providing a direct molecular evidence for the presence of oxygen during electrochemical gas evolution reaction.

\section{Kinetic Analysis of Multistage Oxygen Diffusion at the Electrode-Electrolyte Interface.}

The dynamic extinction spectral evidence for oxygen evolution exhibited different accumulation or dissipation rate of dissolved oxygen on the EEIs, and indicated the presence of multistage oxygen diffusion. We next monitored the extinction voltammetry under different potential scan rate in order to effectively identify the diffusion of dissolved oxygen (Fig. 4a-b). Kinetic measurements at different potential scan rate exhibited a square root of scan ratedependent relationship for Peak b and c (Fig. 4a, inset), indicating the oxygen evolution at the $\mathrm{Cu}$ NPs electrode was restrained by removal of gas products $\left(\mathrm{O}_{2}\right)$ from the EEIs, rather than supply of reactants $\left(\mathrm{OH}^{-}\right)$from bulk electrolyte under strong alkaline conditions. ${ }^{41,42}$ This accumulating inhibition effect was originated from the lower solubility and diffusion coefficient of dissolved oxygen, which was 87 and 2.8 times less than that of hydroxide, respectively. ${ }^{43}$ Similar oxygen transport induced accumulating inhibition effect on OER was also observed at single $\mathrm{CoFe}_{2} \mathrm{O}_{4}$ nanoparticles. ${ }^{44}$ With increasing the scan rate, the resulting oxygen concentration profile change led to linear increasing of the premature oxygen 

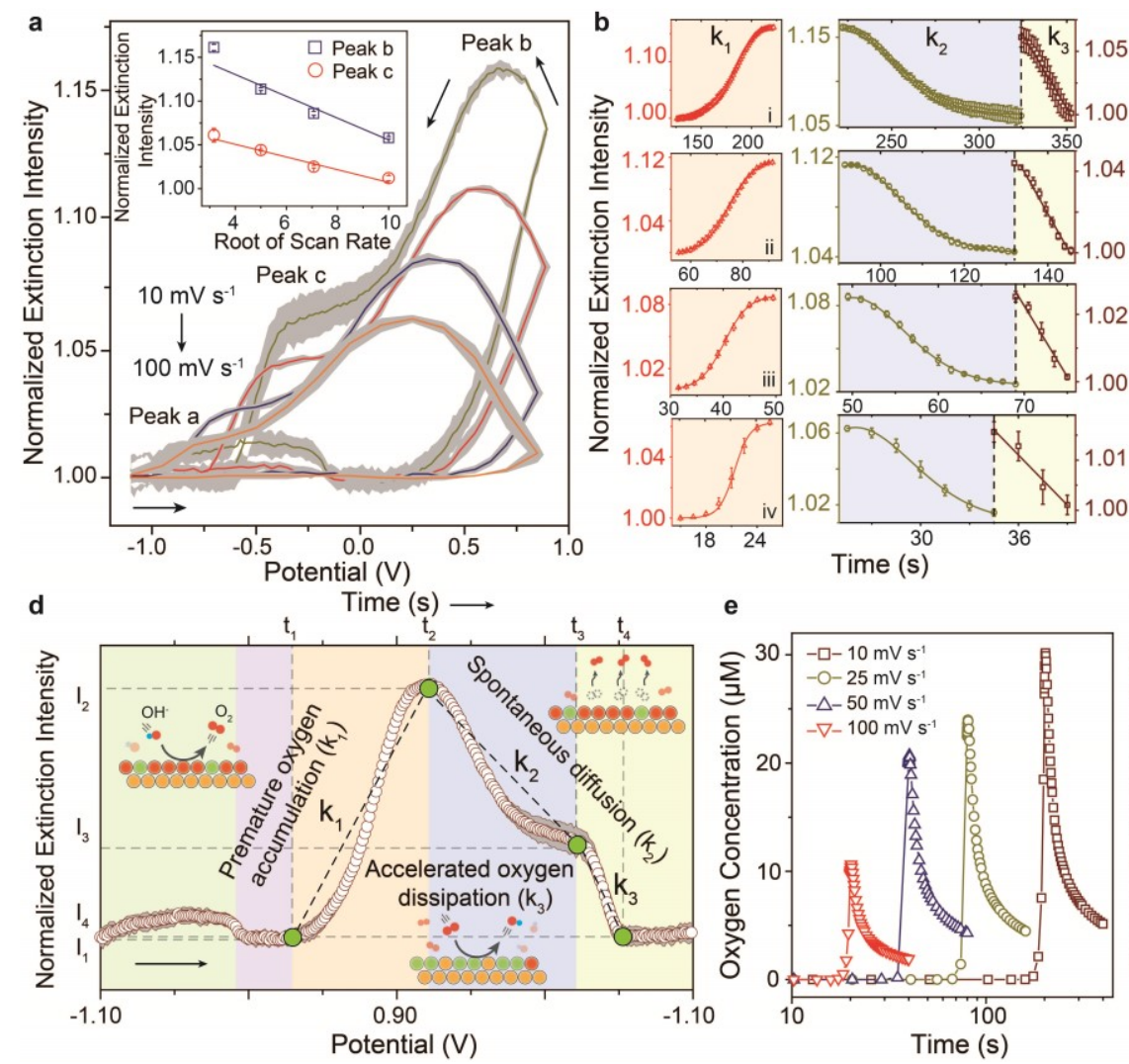

Fig. 4 Kinetic analysis of multistage oxygen diffusion at the EEIs. (a) The scan rate dependent behavior for extinction voltammetry. Inset shows the relationship between extension intensity (peak b and c) and the square root of the scan rate. (b) The extinction intensity evolution during oxygen diffusion and oxygen reduction region at various scan rates $0.01,0.025,0.05$ and $0.10 \mathrm{~V} \mathrm{~s}^{-1}$ (i-iv). (c) The oxygen accumulation rate $k_{l}$ compared with peak $\mathrm{b}(\mathrm{i})$, the oxygen diffusion rate $k_{2}$ (ii), and oxygen reduction rate $k_{3}$ compared with peak $\mathrm{c}$ as a function of the square root of the scan rate (iii). (d) Schematic of apparent oxygen accumulation rate $k_{I}\left(k_{I}=\left|I_{2}-I_{I}\right|\left(t_{2}-t_{1}\right)^{-1}\right)$, oxygen diffusion rate $k_{2}\left(k_{2}=\left|I_{3}-I_{2}\right|\left(t_{3}-t_{2}\right)^{-1}\right)$ and dissipation rate $k_{3}\left(k_{3}=\left|I_{4}-I_{3}\right|\left(t_{4}-t_{3}\right)^{-1}\right)$ during dynamic potential scan. Inset shows the interfacial reactions. (e) Numerical simulation of oxygen concentration evolution on the electrode surface at various potential scan rate $\left(0.01,0.025,0.05\right.$ and $\left.0.10 \mathrm{~V} \mathrm{~s}^{-1}\right)$. (f) Numerical simulation of concentration profiles at various polarization potential corresponding to the peak potential (peak b) at various scan rates $0.01,0.025,0.05$ and $0.10 \mathrm{~V} \mathrm{~s}^{-1}$, respectively (top panel). The comparison between the dependence of oxygen concentration at the EEIs and extinction intensity (peak b) on the square root of the scan rate (bottom panel).

accumulation rate $k_{1}$, diffusion rate $k_{2}$ and nonlinear increasing of accelerated oxygen dissipation rate $k_{3}$ (Fig. $4 \mathrm{c}-\mathrm{d}$ ). By increasing the oxygen accumulation rate $k_{1}$, the resulting oxygen concentration profile changes such that the electrode interface will experience various polarization effects, inducing inhibition and corresponding deterioration in oxygen evolution performance. In contrast, decreasing the oxygen accumulation rate at low scan rate will effectively relieve the influence of accumulation of oxygen on the catalytic performance, and 
explained the increased oxygen concentration at the electrode surface. These findings showed that the oxygen accumulation rate $k_{1}$ was critical in studies that attempted to identify oxygen accumulating inhibition effect on oxygen evolution performance. As increasing the scan rate, a low oxygen concentration at the EEIs was observed due to the severe inhibition on the oxygen evolution induced by the fast oxygen accumulation rate. Despite the low dissolved oxygen concentration on the EEIs, it contributed to the relative fast diffusion rate $k_{2}$ during the sluggish oxygen diffusion region in which insufficient anodic polarization cannot driven the steadily oxygen evolution and thus dominated by the spontaneously dissolved oxygen diffusion. Note that the sharply attenuation of dissolved oxygen was observed as enhancing of cathodic polarization, indicated the presence of accelerated oxygen dissipation near the electrode surface.

The nonlinear increasing of the oxygen dissipation rate $k_{3}$ as increasing of scan rate was explained by the enhanced oxygen reduction rate with gradually increasing coverage of the catalytic $\mathrm{Cu}(\mathrm{OH})_{2}$ layer or submonolayer $\mathrm{Cu}_{2} \mathrm{O}$ surface. The possible electron relay between adjacent copper sites, $\mathrm{Cu}_{2} \mathrm{O}$ and $\mathrm{Cu}(\mathrm{OH})_{2}$, promoted the electron transfer to adsorbed oxygen on the catalytic sites, which was responsible to the enhanced oxygen reduction rate. ${ }^{45}$ The ability to visualize these oxygen evolution profiles was a direct result of our plasmonicenhanced platform that allows in-situ monitoring gas evolution at the EEIs under various polarization, which prevented the artificial interferences in ex-situ measurements. These results suggested that the dissovled oxygen could be adsorbed on the electrotrde interface by noncovalent van der Waals interactions, leading to the blockage of active sites and the restraint of charge-transfer. This multistage diffusion thus revealed how oxygen accumulation inhibited the oxygen evolution performance. 
To further explore the mass transfer of dissolved oxygen and charge transfer at the EEIs, finite element method (FEM) was performed by combining of the Bulter-Volmer equation and Fick's 2nd law (Fig. 4e-f). ${ }^{46}$ The electrocatalytic performances with charging correction decreased as an increasing of potential scan rate, exhibited a similar trend with the extinction measurements (Supplementary Fig. 14 and 22). Meanwhile, the numerical simulated oxygen evolution profile at various scan rates showed consistent oxygen evolution and diffusion behavior compared with in-situ extinction voltammetry, and confirmed the capability for the extinction voltammetry in monitoring oxygen evolution (Fig. 4e). Moreover, concentration contour maps of the oxygen diffusion layer revealed that the diffusion region of dissolved oxygen was more inclined to confined at the electrode surface with a shorter diffusion length $(150 \mu \mathrm{m})$ at a higher scan rate $\left(0.10 \mathrm{~V} \mathrm{~s}^{-1}\right)$, and the thickness of diffusion region increased with decreasing scan rate (Supplementary Fig. 22). Notably, the extinction intensity at peak b showed a good agreement with the simulated oxygen concentration at the EEIs as a square root of the scan rate (Fig. 4f). These results further demonstrated the effectivity of plasmonic single wavelength strategy in revealing details on the interfacial dynamics directed toward oxygen evolution and further supported that mass transport of dissolved oxygen-imposed restriction effects on OER. We proposed that the confinement of dissolved oxygen at the EEIs was responsible for impeding oxygen evolution, leading to a relative higher oxygen concentration polarization as well as conjectural local heterogeneous supersaturated regions readily for oxygen bubbles nucleation and growth.

Improving OER Performance through Programmable Potential Scan. Gradual enrichment of dissolved oxygen leads to an increased concentration polarization and a blockage of 


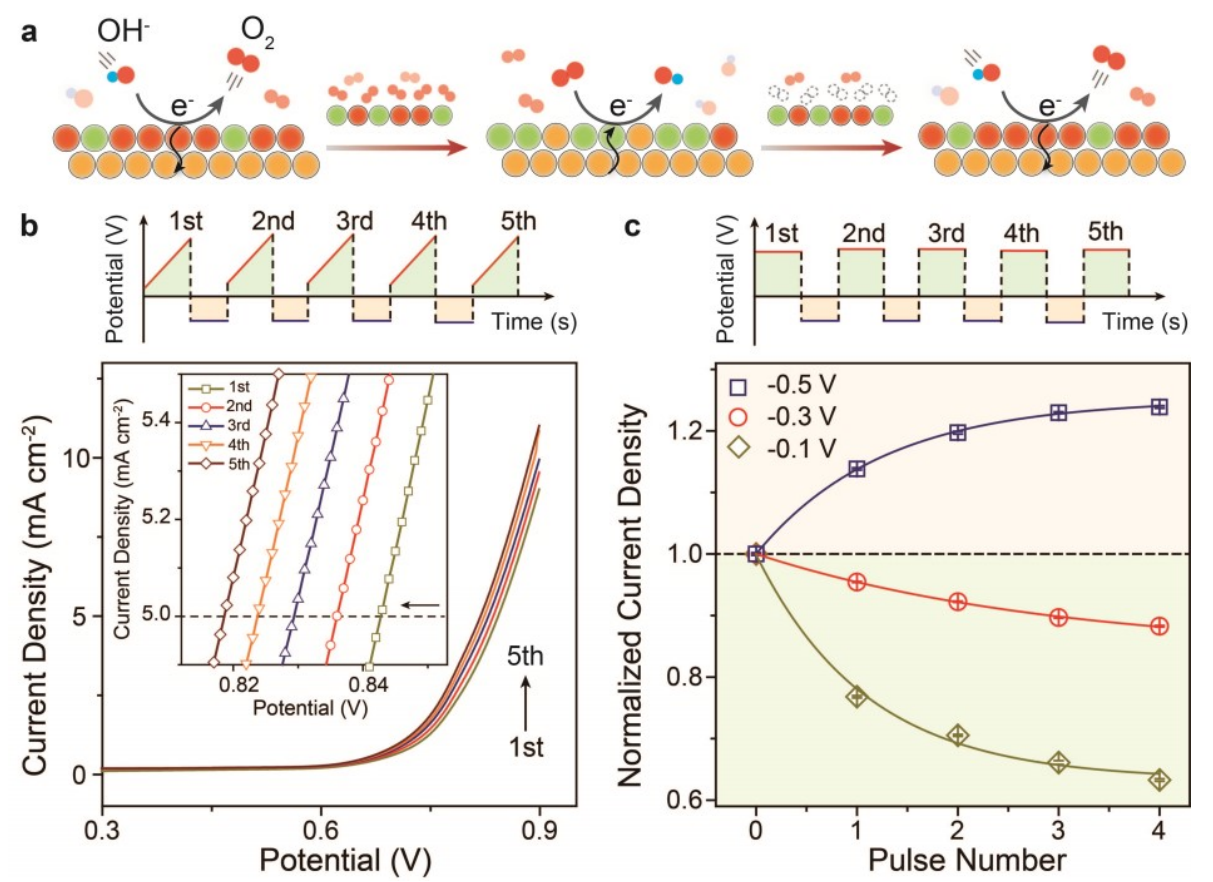

Fig. 5 Programmable potential scan strategy to eliminate accumulated interfacial dissolved oxygen at the EEIs. (a) Schematic illustration of reduction potential pulse accelerating interfacial oxygen species remove from the electrode surface. (b) Linear sweep voltammetry curves of $\mathrm{Cu}$ electrode combined with reduction potential multi-pulse $(-0.50 \mathrm{~V})$ in $0.10 \mathrm{M} \mathrm{KOH}$ solution at a scan rate of $0.01 \mathrm{~V} \mathrm{~s}^{-1}$. Inset shows cathodic shift of the oxygen evolution potential as consecutive reduction potential pulses $(-0.50 \mathrm{~V})$. (c) Mean chronoamperometry current density at $0.70 \mathrm{~V}$ as a function of reduction potential multi-pulse $(-0.50,-0.30$ and $-0.10 \mathrm{~V})$ normalized by the value of the original current density (1st scan). Schematic of the programmed potential scan strategy shown in upper panel in (b) and (c), respectively.

interfacial active sites at the electrode surface. The precise recognition of multistage oxygen diffusion enables us to accelerate the accumulated dissolved oxygen remove from the electrode surface without forced convection, further alleviate the dissolved oxygen concentration polarization and improve electrocatalytic performance. We thereby established a programmable potential scan strategy to remove the adsorbed interfacial dissolved oxygen species (Fig. 5a) in order to effectively eliminate these adverse effects. In brief, the program able cathodic polarization facilitated the reduction of nonspecific adsorption of hydrated oxygen located in the outer Helmholtz plane and increased the accessibility of interfacial hydroxide. ${ }^{47}$ With the ability of identifying spontaneous diffusion $(0.67 \mathrm{~V}$ to $-0.35 \mathrm{~V})$ and 
accelerated oxygen dissipation $(-0.35 \mathrm{~V}$ to $-0.63 \mathrm{~V})$ region, reduction potential multi-pulse at $-0.50 \mathrm{~V}$, and -0.30 and $-0.10 \mathrm{~V}$ were chosen as control potentials to confirm the effectiveness of proposed concept. The overpotential negatively shifted $24 \mathrm{mV}$ under reduction potential multi-pulse $(-0.50 \mathrm{~V})$ compared to the positive shift $c a .12 \mathrm{mV}$ at both -0.30 and $0.10 \mathrm{~V}$, indicating the effective alleviation of polarization potential by removing the adsorption dissolve oxygen species (Fig. 5b and Supplementary Fig. 23). Notably, the prominent enhancement $24.1 \%$ of current density at $0.70 \mathrm{~V}$ under reduction potential multi-pulse $(-0.50$ $\mathrm{V}$ ) in contrast to the attenuation $11.8 \%$ and $36.7 \%$ at -0.30 and $-0.10 \mathrm{~V}$, respectively, originated from the increased exposure of interfacial adsorbed hydroxides, a consequence of removing dissolved oxygen from the electrode surface (Fig. 5c and Supplementary Fig. 24). These results confirmed the validity of the electric field-induced elimination of interfacial dissolved oxygen concept on promoting OER performance, and suggested a general principle for the improvement of fuel cell reactions at the EEIs.

\section{Discussion}

In summary, we real-time monitored oxygen diffusion at the EEIs by using in-situ extinction spectroelectrochemistry and mass spectrometry. The combination of in-situ techniques, not only provided an unprecedented understanding toward the dynamic behavior of catalytic interface without the interference of non-Faradaic effects, but also revealed the oxygen accumulation at the EEIs as a limiting factor on oxygen evolution reaction and corroborated the presence of multistage diffusion behavior of dissolved oxygen, which cannot be precisely identified in conventional electrochemical methods. This was specially important for providing 
a direct strategy for gas monitoring without introducing any chemical recognition components and facilitate electrocatalytic performance as this could be translated into more complex gas evolving electrochemical systems, such as those generate further gas species $\left(\mathrm{H}_{2}, \mathrm{CO}_{2}\right.$ and $\left.\mathrm{N}_{2}\right)$ through more complicated catalytic interfacial chemistry. Our strategy can be further general for readily evaluation the non-plasmonic electrocatalysts performance by coupling advanced plasmonic antenna, thus allowing efficient development of desirable catalysts and leading to next generation functional energy device.

\section{Methods}

Electrochemical Measurements. A CHI660E electrochemical station (CH Instruments, Shanghai, China) was used to validate occurring of gas evolution reaction in a microelectrochemical cell. Electrochemical measurements were performed in a conventional onecompartment cell in conjunction with a standard three-electrode system and the microelectrochemical cell. In a conventional cell, the electrochemical seed-mediated growth of $\mathrm{Cu}$ NPs on the ITO electrode was performed according to the above procedures. Linear sweep voltammetry (LSV) was performed at a scan rate of $0.01 \mathrm{~V} \mathrm{~s}^{-1}$ to qualitatively evaluate the catalytic activity for oxygen evolution and define the onset potential of surface gas evolution applied to compare the performance in monitoring gas-involved electrochemical reaction using various in-situ spectroelectrochemistry methods.

Electrochemical measurements coupled with in-situ extinction spectroscopy were performed in a homemade three-electrode micro-electrochemical cell with a Pt mesh counter electrode $(\mathrm{CE})$ and a Pt wire quasi-reference electrode (QRE). The extinction spectra were recorded in a spectral range from 390 to $1000 \mathrm{~nm}$ during potentiostatic polarization measurements. 
Electrochemical extinction measurements were conducted at steady-state conditions, and the as-prepared $\mathrm{Cu}$ NPs/ITO electrode was allowed to equilibrate at each potential for at least 2 min prior to extinction spectral acquisition. A total of 5 scans were averaged for 1 spectrum, which requiring an accumulation time of $90 \mathrm{~s}$. We began by applying a constant potential of $1.10 \mathrm{~V} v s$. Pt and measuring the extinction spectrum over the course of $3.5 \mathrm{~min}$, then applied a more positive potential and re-measured the extinction spectrum for another $3.5 \mathrm{~min}$ continuing in a step-wise fashion up to $0.90 \mathrm{~V}$. The evolution of the extinction spectra was tracked as a function of potential.

Kinetic experiments for in-situ probing the oxygen evolution reaction were performed by monitoring the intensity located at extinction peak (recognized from the extinction spectra of each $\mathrm{Cu}$ NPs/ITO electrode) over time as a particular voltage in a stepped chronoamperometric mode. On the basis of cyclic voltammetry curves in a $0.1 \mathrm{M} \mathrm{KNO}_{3}$ solution containing 5.00 $\mathrm{mM} \mathrm{K}_{3}\left[\mathrm{Fe}(\mathrm{CN})_{6}\right]$ (Figure $\mathrm{S} 1$ ), the $\Delta E_{0}{ }^{1 / 2}$ was calculated as $0.286 \mathrm{~V}$ between using Pt QRE and saturated calomel electrode (SCE). In the micro-electrochemical cell, real time monitoring of gas evolution reaction during cyclic voltammetry in potential range from -1.10 to $0.90 \mathrm{~V}$ ( $v s$. $\mathrm{Pt}$ ) at different scan rates was investigated by in-situ extinction electrochemical measurements. The extinction spectra assigned to fluctuations of the electrode-electrolyte interface on the $\mathrm{Cu}$ NPs/ITO electrode during dynamic potential scan were collected synchronously with corresponding electrochemical information. The electrolyte solution was bubbled with $\mathrm{N}_{2}$ gas for 20 min prior to eject into the micro-electrochemical cell.

Rotating ring-disk electrode (RRDE) electrochemical measurements were performed using either a bipotentiostat model CHI760D (CH Instruments, Shanghai, China) and a Pine model 
AFCBP1 bipotentiostat (RRDE). During RRDE cyclic voltammogram measurements, the working electrode used a fixed-disk RRDE with glass carbon disk OD $5.61 \mathrm{~mm}$, ring OD 7.92 $\mathrm{mm}$ and ring ID $6.25 \mathrm{~mm}$, and PTFE shroud $15.0 \mathrm{~mm}$. The apparent RRDE collection efficiency $(37.0 \%$ at $1600 \mathrm{rpm})$ was estimated from the ring and disk limiting current ratios in $0.10 \mathrm{M} \mathrm{KOH}$ and $5 \mathrm{mM} \mathrm{K}_{3}\left[\mathrm{Fe}(\mathrm{CN})_{6}\right]$ solution. The various collection potential $(-0.10,-0.50$, -0.70 and $-0.90 \mathrm{~V} v s \mathrm{Pt}$ ) were evaluated in RRDE experiments for oxygen reduction reaction. The fabrication of $\mathrm{Cu}$ NPs/GC electrode follows the similar procedures illustrated in above section. The counter and quasi-reference electrode in all RRDE experiments was both Pt wire electrode. The resistance values estimated from electrochemical impedance measurements ranged from 25 to $36 \mathrm{Ohm}$. All experiments were performed at room temperature $25 \pm 2{ }^{\circ} \mathrm{C}$.

Instruments. AFM measurements were performed on a Bioscope Resolve AFM (Bruker Corp., USA) under tapping mode with a scan rate of $1.0 \mathrm{~Hz}$ frequency. AFM images with a resolution of $512 \times 512$ pixels were acquired using silicon cantilevers with a normal spring constant of $0.35 \mathrm{~N} \mathrm{~m}^{-1}$. All recorded images were treated with "Flatten" function prior to analysis in NanoScope Analysis software (version 1.60). UV-Vis spectra were recorded on a UV-2600 spectrophotometer (Shimadzu, Japan).

ToF-SIMS measurements were performed using a ToF-SIMS V (ION-TOF GmbH, Münster, Germany) mass spectrometer equipped with a time-of-flight analyzer of a reflectron type. In brief, a $30 \mathrm{keV} \mathrm{Bi}_{3}{ }^{+}$primary ion beam was used at $10 \mathrm{kHz}$ frequency with a pulsed beam current of $0.36 \mathrm{pA}$, and it was focused to be $\sim 450 \mathrm{~nm}$ diameter in fast imaging mode. All ion image resolution was $512 \times 512$ pixels. The positive and negative mass spectra were calibrated by $\mathrm{CH}_{3}{ }^{+}, \mathrm{H}_{3} \mathrm{O}^{+}$and $\mathrm{NH}_{4}^{+}$and $\mathrm{C}^{-}, \mathrm{OH}^{-}$and $\mathrm{C}_{2}^{-}$, respectively. The vacuum pressure in the main 
chamber during the measurements was below $3 \times 10^{-9} \mathrm{mbar}$.

X-ray diffraction (XRD) patterns were collected on a Bruker D8-ADVANCE X-ray diffractometer with DteX Ultra 250 detector and copper target using monochromatic $\mathrm{Cu} \mathrm{K}_{\alpha}$ radiation $(\lambda=1: 5406 \AA)$. The range of diffraction (Bragg) angles were measured within the scan range from $10^{\circ}$ to $80^{\circ}$ with a step angle of $0.02^{\circ}$ and a scan rate of $20^{\circ} \min ^{-1}$ at $40 \mathrm{KV}, 30$ $\mathrm{mA}$. The incident Soller slit and length-limiting slit was set as $2.5^{\circ}$ and $5 \mathrm{~mm}$, respectively.

Field Emission transmission electron microscope (TEM) images were collected on JEOL2100F (Japan) equipped with an energy dispersive X-ray detector and operated at voltage 200 $\mathrm{kV}$. High-resolution TEM images, selected area electron diffraction (SAED) patterns and relative elemental mapping were carried out during HRTEM measurements. For TEM characterization, the electrochemical deposited $\mathrm{Cu}$ nanoparticles were gently scraped by a steel blade from the ITO electrode and subsequently dispersed in anhydrous acetonitrile solution by sonication (20 s). Then, a droplet of the suspension with $20 \mu \mathrm{L}$ was gently dropped onto a carbon coated-copper grid (300 mesh), allowing the solvent to evaporate prior to imaging.

\section{Data availability}

The data that support this paper and other findings of this study are available from the corresponding authors upon reasonable request.

\section{References}

1. Velasco-Velez J.-J. et al., The structure of interfacial water on gold electrodes studied by xray absorption spectroscopy. Science 346, 831 (2014).

2. Li C.-Y. et al., In situ probing electrified interfacial water structures at atomically flat surfaces. Nat. Mater. 18, 697-701 (2019).

3. Zhou Y. et al., Real-time mass spectrometric characterization of the solid-electrolyte 
interphase of a lithium-ion battery. Nat. Nanotechnol. 15, 224-230 (2020).

4. Tao H. B. et al., Revealing energetics of surface oxygen redox from kinetic fingerprint in oxygen electrocatalysis. J. Am. Chem. Soc. 141, 13803-13811 (2019).

5. Suen N.-T. et al., Electrocatalysis for the oxygen evolution reaction: recent development and future perspectives. Chem. Soc. Rev. 46, 337-365 (2017).

6. Seitz L. C. et al., A highly active and stable $\mathrm{IrO}_{x} / \mathrm{SrIrO}_{3}$ catalyst for the oxygen evolution reaction. Science 353, 1011 (2016).

7. Albinsson D. et al., Operando detection of single nanoparticle activity dynamics inside a model pore catalyst material. Sci. Adv. 6, eaba7678 (2020).

8. Jiang H., He Q., Zhang Y., Song L., Structural self-reconstruction of catalysts in electrocatalysis. Acc. Chem. Res. 51, 2968-2977 (2018).

9. Zhu Y., Wang J., Chu H., Chu Y.-C., Chen H. M., In situ/operando studies for designing next-generation electrocatalysts. ACS Energy Lett. 5, 1281-1291 (2020).

10. Gao J. et al., Breaking long-range order in Iridium oxide by alkali ion for efficient water oxidation. J. Am. Chem. Soc. 141, 3014-3023 (2019).

11. Li J., Peng Z., Wang E., Tackling grand challenges of the 21 st century with electroanalytical chemistry. J. Am. Chem. Soc. 140, 10629-10638 (2018).

12. Fabbri E. et al., Dynamic surface self-reconstruction is the key of highly active perovskite nano-electrocatalysts for water splitting. Nat. Mater. 16, 925-931 (2017).

13. Tung C.-W. et al., Reversible adapting layer produces robust single-crystal electrocatalyst for oxygen evolution. Nat. Commun. 6, 8106 (2015).

14. Zheng W., Liu M., Lee L. Y. S., Electrochemical instability of metal-organic frameworks: in situ spectroelectrochemical investigation of the real active sites. ACS Catal. 10, 81-92 (2020).

15. Wang J.-G. et al., Direct Molecular evidence of proton transfer and mass dynamics at the electrode-electrolyte interface. J. Phys. Chem. Lett. 10, 251-258 (2019)

16. Peng Z., Freunberger S. A., Chen Y., Bruce P. G., A Reversible and Higher-Rate $\mathrm{LiO}_{2}$ Battery. Science 337, 563 (2012).

17. Xiang Y. et al., Visualizing the growth process of sodium microstructures in sodium 
batteries by in-situ 23Na MRI and NMR spectroscopy. Nat. Nanotechnol. 15, 883-890 (2020).

18. Wang W., Imaging the chemical activity of single nanoparticles with optical microscopy. Chem. Soc. Rev 47, 2485-2508 (2018).

19. Wilson A. J., Devasia D., Jain P. K., Nanoscale optical imaging in chemistry. Chem. Soc. $\operatorname{Rev~49,~6087-6112~(2020).~}$

20. Li W. et al., Single-molecular catalysis identifying activation energy of the intermediate product and rate-limiting step in plasmonic photocatalysis. Nano Lett. 20, 2507-2513 (2020).

21. Su H.-S. et al., Probing the local generation and diffusion of active oxygen species on a $\mathrm{Pd} / \mathrm{Au}$ bimetallic surface by tip-enhanced Raman spectroscopy. J. Am. Chem. Soc. 142, 1341-1347 (2020).

22. Liu S. et al., In Situ Plasmonic Nanospectroscopy of the CO oxidation reaction over single Pt nanoparticles. ACS Nano 13, 6090-6100 (2019).

23. Wang J.-G., Jing C., Long Y.-T., "Single-Nanoparticle Plasmonic Spectroelectrochemistry" in Frontiers of Plasmon Enhanced Spectroscopy Volume 2. (American Chemical Society, 2016), vol. 1246, chap. 4, pp. 57-96.

24. Hoener B. S. et al., Plasmonic sensing and control of single-nanoparticle electrochemistry. Chem 4, 1560-1585 (2018).

25. Dong J.-C. et al., In situ Raman spectroscopic evidence for oxygen reduction reaction intermediates at platinum single-crystal surfaces. Nat. Energy 4, 60-67 (2019).

26. Chen J. et al., Measuring the activation energy barrier for the nucleation of single nanosized vapor bubbles. Proc. Natl. Acad. Sci. U.S.A. 116, 12678 (2019).

27. Li S. et al., Nanobubbles: An effective way to study gas-generating catalysis on a single nanoparticle. J. Am. Chem. Soc. 139, 14277-14284 (2017).

28. Jin Y. et al., In operando plasmonic monitoring of electrochemical evolution of lithium metal. Proc. Natl. Acad. Sci. U.S.A. 115, 11168 (2018).

29. Li K. et al., DNA-Directed Assembly of Gold Nanohalo for Quantitative Plasmonic Imaging of Single-Particle Catalysis. J. Am. Chem. Soc. 137, $4292-4295$ (2015). 
30. Wang P. et al., Plasmonic metamaterials for nanochemistry and sensing. Acc. Chem. Res. 52, 3018-3028 (2019).

31. Ueda M. et al., Double-pulse technique as an electrochemical tool for controlling the preparation of metallic nanoparticles. Electrochim. Acta 48, 377-386 (2002).

32. Pérez-González O. et al., Optical spectroscopy of conductive junctions in plasmonic cavities. Nano Lett. 10, 3090-3095 (2010).

33. Schnell M. et al., Controlling the near-field oscillations of loaded plasmonic nanoantennas. Nat. Photonics 3, 287-291 (2009).

34. Dong S., Zhang K., Yu Z., Fan J. A., Electrochemically programmable plasmonic antennas. ACS Nano 10, 6716-6724 (2016).

35. Gerislioglu B. et al., Monolithic metal dimer-on-film structure: new plasmonic properties introduced by the underlying metal. Nano Lett. 20, 2087-2093 (2020).

36. Gargiulo J., Berté R., Li Y., Maier S. A., Cortés E., From optical to chemical hot spots in plasmonics. Acc. Chem. Res. 52, 2525-2535 (2019).

37. Deng Y., Handoko A. D., Du Y., Xi S., Yeo B. S., In situ Raman spectroscopy of copper and copper oxide surfaces during electrochemical oxygen evolution reaction: identification of CuIII oxides as catalytically active species. ACS Catal. 6, 2473-2481 (2016).

38. Bodappa N. et al., Early stages of electrochemical oxidation of $\mathrm{Cu}(111)$ and polycrystalline $\mathrm{Cu}$ surfaces revealed by in situ Raman spectroscopy. J. Am. Chem. Soc. 141, 12192-12196 (2019).

39. Zhan C., Liu B.-W., Tian Z.-Q., Ren B., Determining the interfacial refractive index via ultrasensitive plasmonic sensors. J. Am. Chem. Soc. 142, 10905-10909 (2020).

40. Esposito D. V., Membrane-Coated Electrocatalysts—an alternative approach to achieving stable and tunable electrocatalysis. ACS Catal. 8, 457-465 (2018).

41. Molina A., González J., Laborda E., Compton R. G., On the meaning of the diffusion layer thickness for slow electrode reactions. Phys. Chem. Chem. Phys. 15, 2381-2388 (2013).

42. Velasco J. G., On the dependence of the Nernst diffusion layer thickness on potential and sweep rate for reversible and of the thickness of the charge transfer layer for irreversible processes studied by application of the linear potential sweep method. Electrochim. Acta 
51, 2971-2976 (2006).

43. Davis R. E., Horvath G. L., Tobias C. W., The solubility and diffusion coefficient of oxygen in potassium hydroxide solutions. Electrochim. Acta 12, 287-297 (1967).

44. El Arrassi A. et al., Intrinsic activity of oxygen evolution catalysts probed at single $\mathrm{CoFe}_{2} \mathrm{O}_{4}$ nanoparticles. J. Am. Chem. Soc. 141, 9197-9201 (2019).

45. King F., Quinn M. J., Litke C. D., Oxygen reduction on copper in neutral NaCl solution. J. Electroanal. Chem. 385, 45-55 (1995).

46. Wang J.-G., Hua X., Xia H.-L., Long Y.-T., Pore confined liquid-vacuum interface for charge transfer study in an electrochemical process. Anal. Chem. 91, 3195-3198 (2019).

47. Strmcnik D. et al., The role of non-covalent interactions in electrocatalytic fuel-cell reactions on platinum. Nat. Chem. 1, 466-472 (2009). 


\section{Acknowledgements}

This work was supported by the National Natural Science Foundation of China $(21675166$, 21874046, 21902050), National Postdoctoral Program for Innovative Talents (BX20190118), Shanghai Post-Doctoral Excellence Program (2019220), Shanghai Municipal Commission for Science and Technology (19JC1411800), the Fundamental Research Funds for the Central Universities and the original innovation project from 0 to 1 of the Basic Frontier Scientific Research Program of the Chinese Academy of Sciences (ZDBS-LY-JSC010).

\section{Author contributions}

J.-G. W. and D. L. designed research; J.-G. W. and D. L. performed research; J.-G. W., L. L., Y. S., L. S., J. X., Y. T. and D. L. analyzed data; J.-G. W. and D. L. wrote the paper.

\section{Corresponding author}

Di Li

Email: dli@chem.ecnu.edu.cn;

ORCID: D. L.: 0000-0003-1674-0110

\section{Competing interests}

The authors declare no competing interests.

\section{Additional information}

Supplementary information is available for this paper. 


\section{Figures}

a
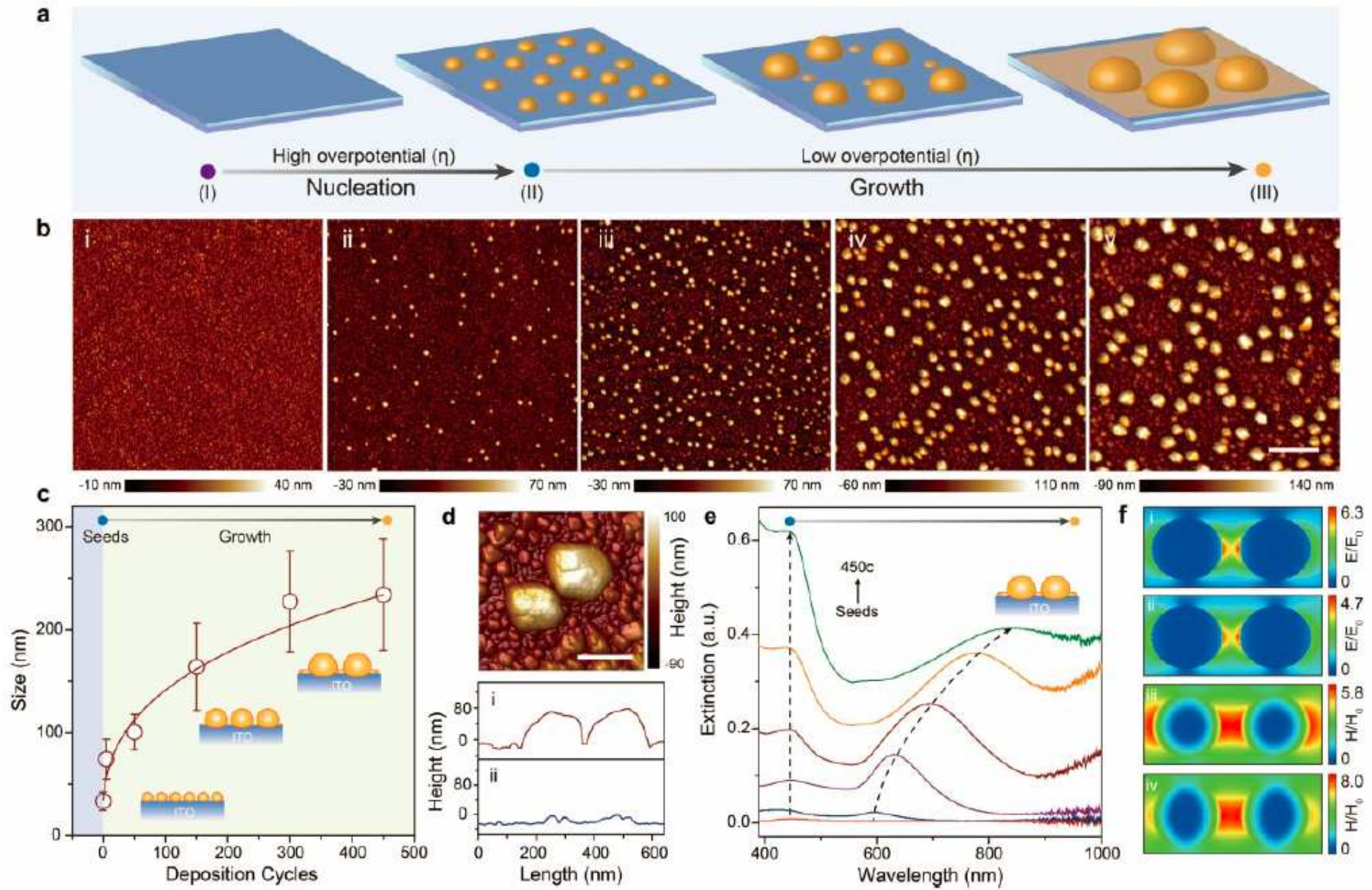

\section{Figure 1}

Seed-mediated electrodeposition of plasmonic Cu nanoparticles. (a) Schematic illustrating the electrochemical seed-mediated growth strategy. (b) AFM images of Cu seeds (i) and Cu NPs at different deposition cycles (ii 5, iii 50, iv 150 and v 300 cycles) (scale bar: $1 \mu \mathrm{m}$ ). (c) The correlation between $\mathrm{Cu}$ NPs size and deposition cycles. (d) High-resolution AFM image of Cu NPs (top, 150 cycles, scale bar: 200 $\mathrm{nm}$ ) and linear scan across Cu NPs dimmer (i) and nearby regions (ii) (bottom). (e) Ex-situ extinction spectra of Cu seeds and Cu NPs at different deposition cycles (5, 50, 150, 300, 450 cycles). (f) Top and side-view of electric ( $\mathrm{i}$ and ii) and magnetic (iii and iv) field snapshots for the near field confinement at the plasmonic resonance wavelengths (i, iii $450 \mathrm{~nm}$ and ii, iv $690 \mathrm{~nm}$ ). 

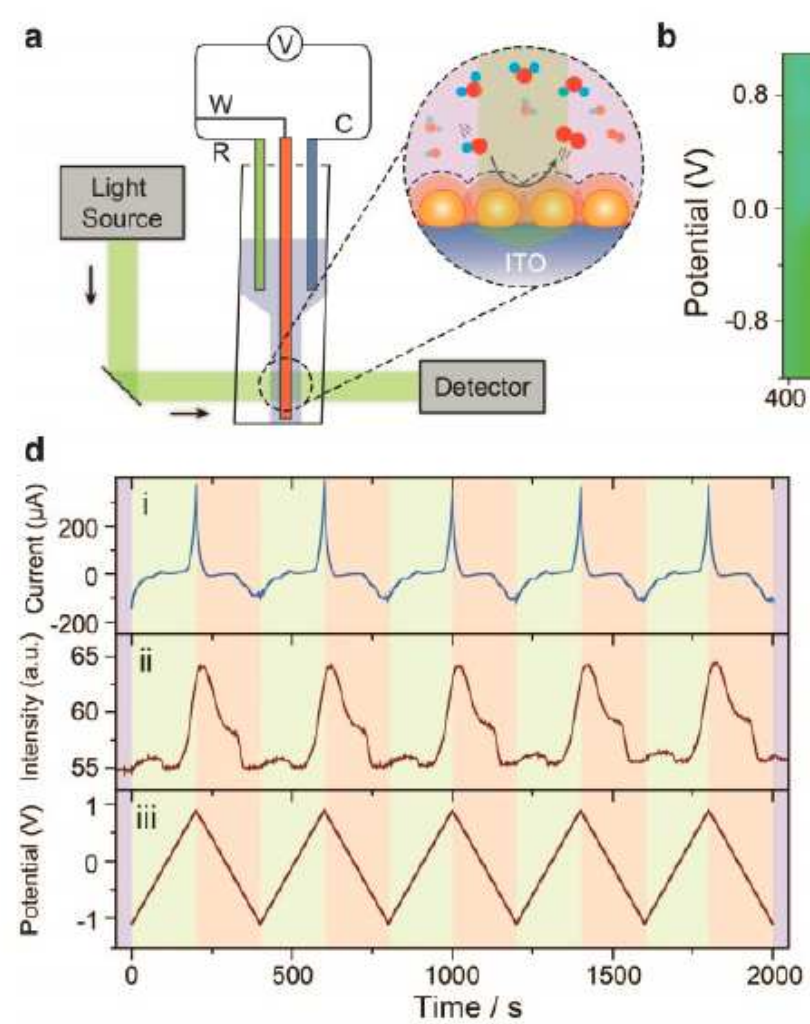

b

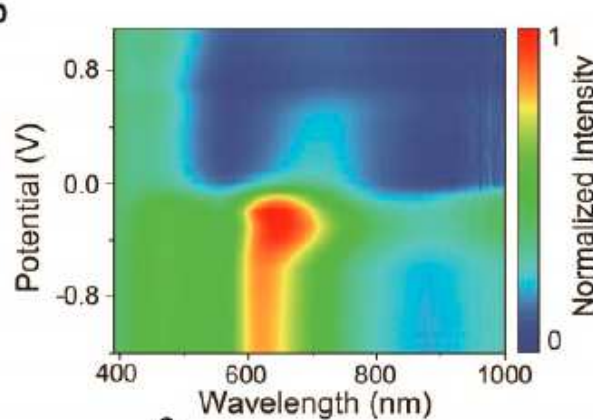

$e^{\text {}}$

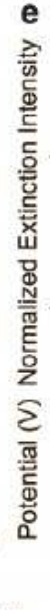

III

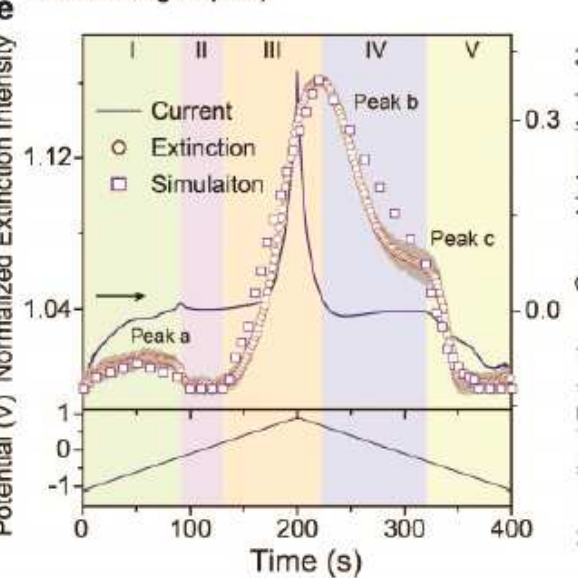

IV

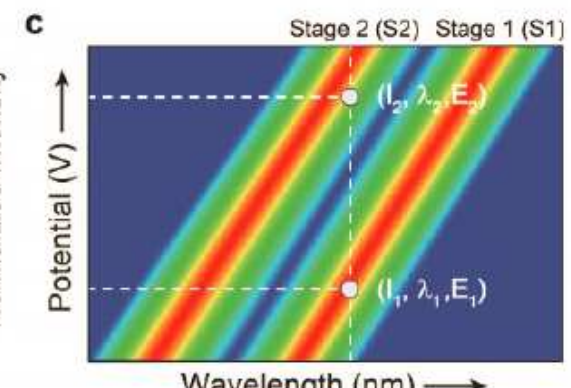

Wavelength $(\mathrm{nm}) \longrightarrow$

g
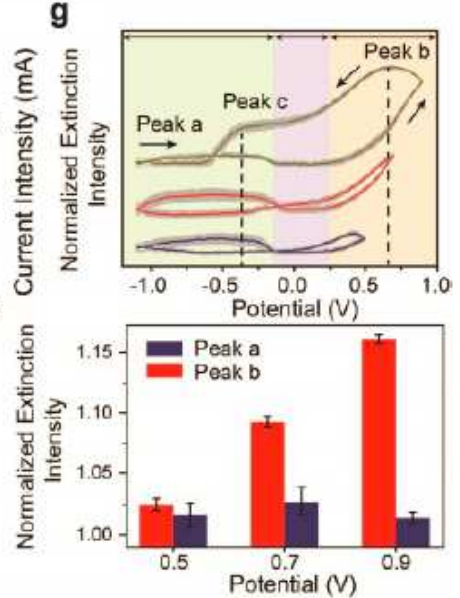

$\mathrm{V}$
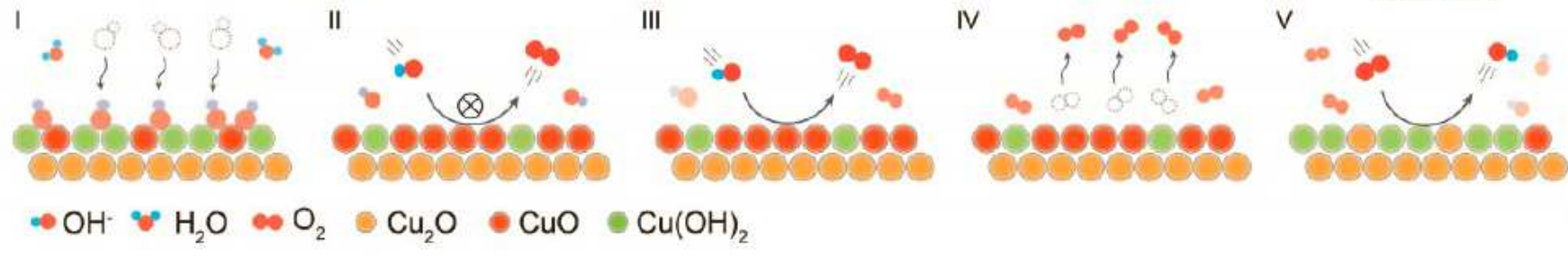

\section{Figure 2}

Plasmonic monitoring oxygen evolution reaction. (a) Experimental setup of in-situ electrochemical extinction microscopy. (b) The normalized dynamic extinction intensity (contour plots) of a Cu NPs electrode under steady-state anodic polarization. (c) Schematic illustration of the plasmonic singlewavelength strategy for real-time monitoring OER at the EEls. (d) The consecutive electrochemical current (i) during OER and synchronous extinction signal (ii) under dynamic potential scan (iii). (e) The representative dynamic extinction trajectory (o), current curve (-) and FDTD simulation ( $\mathbb{(})$ for OER on a plasmonic Cu NPs electrode scanned from -1.10 to $0.90 \mathrm{~V}$ at a scan rate $0.01 \mathrm{~V} \mathrm{~s}-1$. (f) Schematic illustration of the interfacial reaction mechanism during dynamic potential scan. (g) The extinction voltammetry with different terminational potential $(0.50,0.70,0.90 \mathrm{~V})$ at a scan rate of $0.01 \mathrm{~V} \mathrm{~s}-1$ (top panel). The dark region represents the standard error during consecutive five scan. The potential dependent of extinction intensity for peak $a$ and $b$ (bottom panel). 

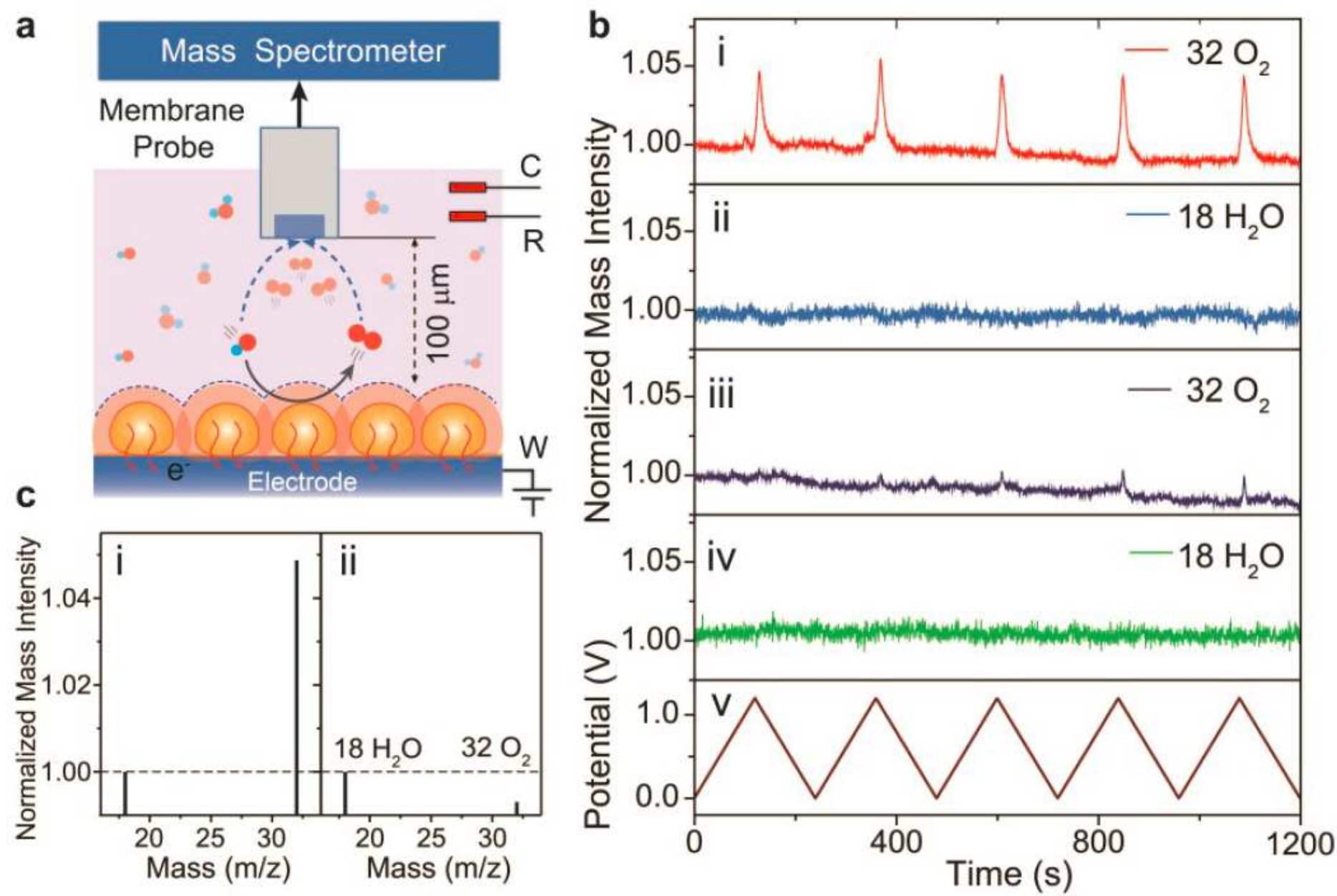

\section{Figure 3}

Potential dependent mass spectrometry of dissolved oxygen. (a) Schematic of in-situ differential electrochemical mass spectrometry. (b) Dynamic mass intensity of 02 and $\mathrm{H} 2 \mathrm{O}$ analyzed by in-situ DEMS during cyclic voltammetry in the potential range from 0 to $1.10 \mathrm{~V}$ on working electrode $\mathrm{Cu} /$ glass carbon-disc (i, ii) and glass carbon-disc (iii) and (iv), respectively, in a homemade micro-electrochemical cell in $0.1 \mathrm{M} \mathrm{KOH}$ solution, at a scan rate of $0.01 \mathrm{~V} \mathrm{~s}-1$. (c) The mass spectra during dynamic potential scan on the working electrode Cu/glass carbon-disc (i) and glass carbon-disc (ii). 

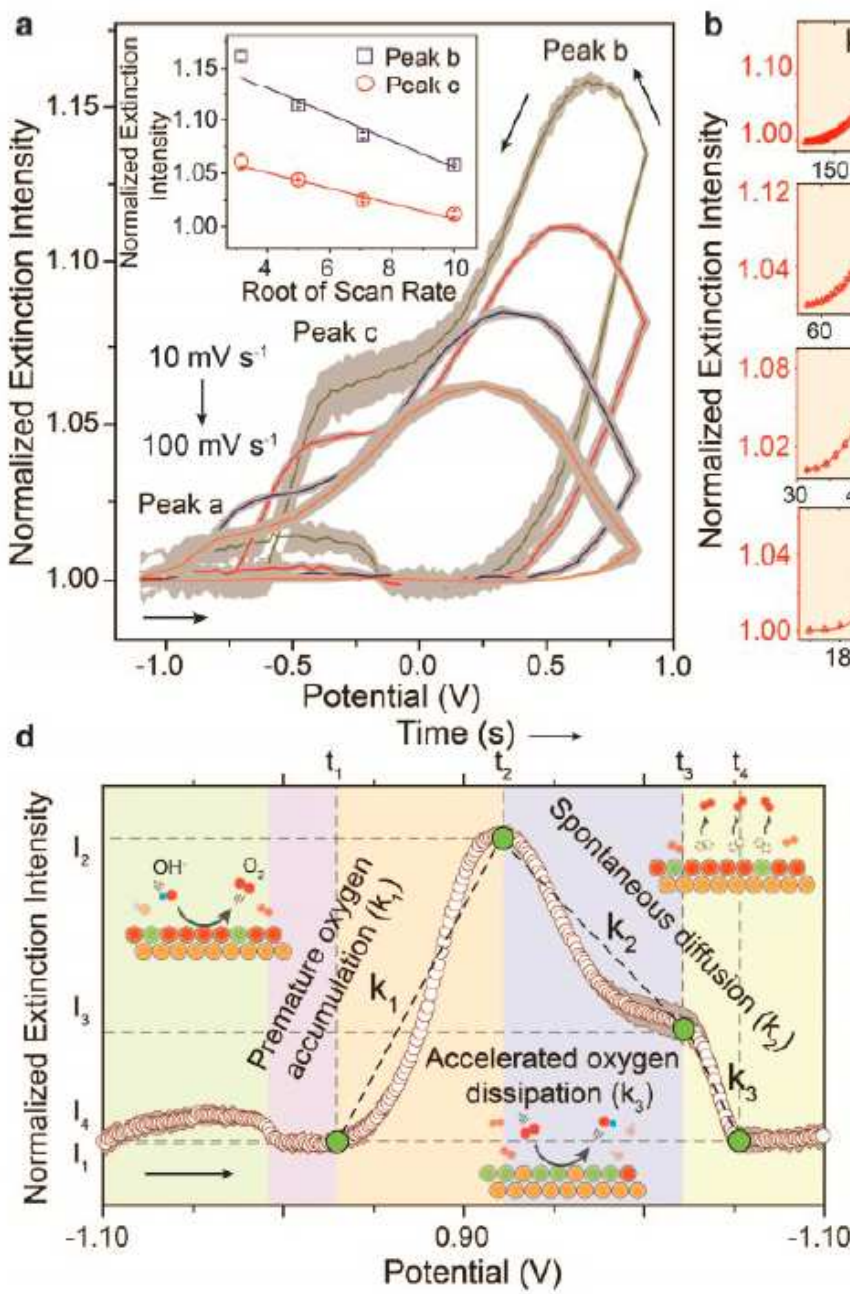

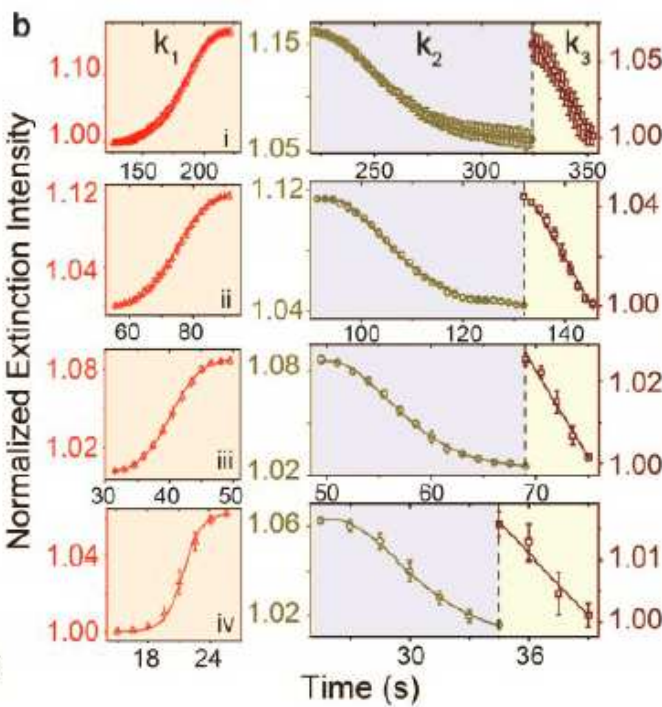

e

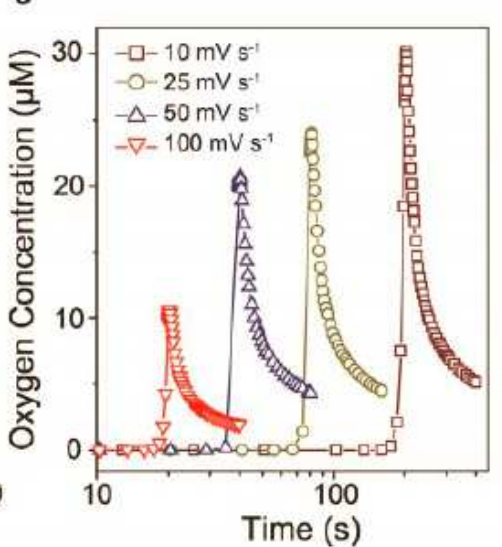

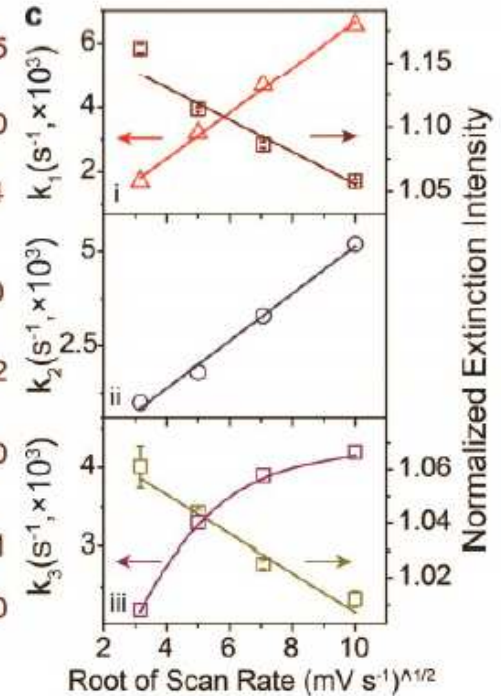

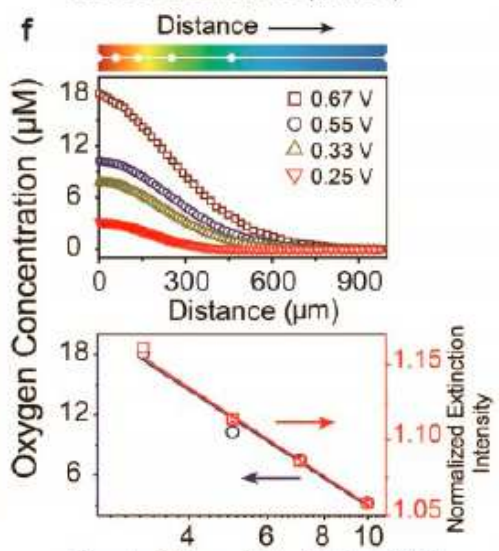

Root of Scan Rate $\left(\mathrm{mV} \mathrm{s}^{-1}\right)^{n+1 / 2}$

\section{Figure 4}

Kinetic analysis of multistage oxygen diffusion at the EEls. (a) The scan rate dependent behavior for extinction voltammetry. Inset shows the relationship between extension intensity (peak b and c) and the square root of the scan rate. (b) The extinction intensity evolution during oxygen diffusion and oxygen reduction region at various scan rates $0.01,0.025,0.05$ and $0.10 \mathrm{~V} \mathrm{~s}-1$ (i-iv). (c) The oxygen accumulation rate $k 1$ compared with peak $b$ (i), the oxygen diffusion rate $k 2$ (ii), and oxygen reduction rate $k 3$ compared with peak $\mathrm{c}$ as a function of the square root of the scan rate (iii). (d) Schematic of apparent oxygen

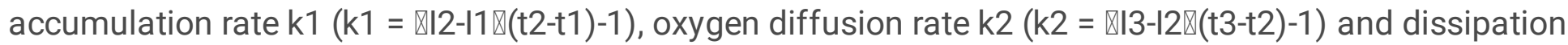

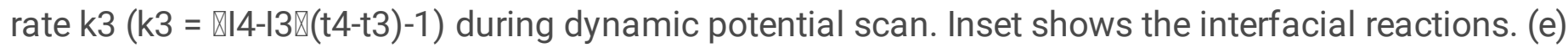
Numerical simulation of oxygen concentration evolution on the electrode surface at various potential scan rate $(0.01,0.025,0.05$ and $0.10 \mathrm{~V} \mathrm{~s}-1)$. (f) Numerical simulation of concentration profiles at various polarization potential corresponding to the peak potential (peak $b$ ) at various scan rates $0.01,0.025,0.05$ and $0.10 \mathrm{~V} \mathrm{~s}-1$, respectively (top panel). The comparison between the dependence of oxygen concentration at the EEls and extinction intensity (peak b) on the square root of the scan rate (bottom panel). 

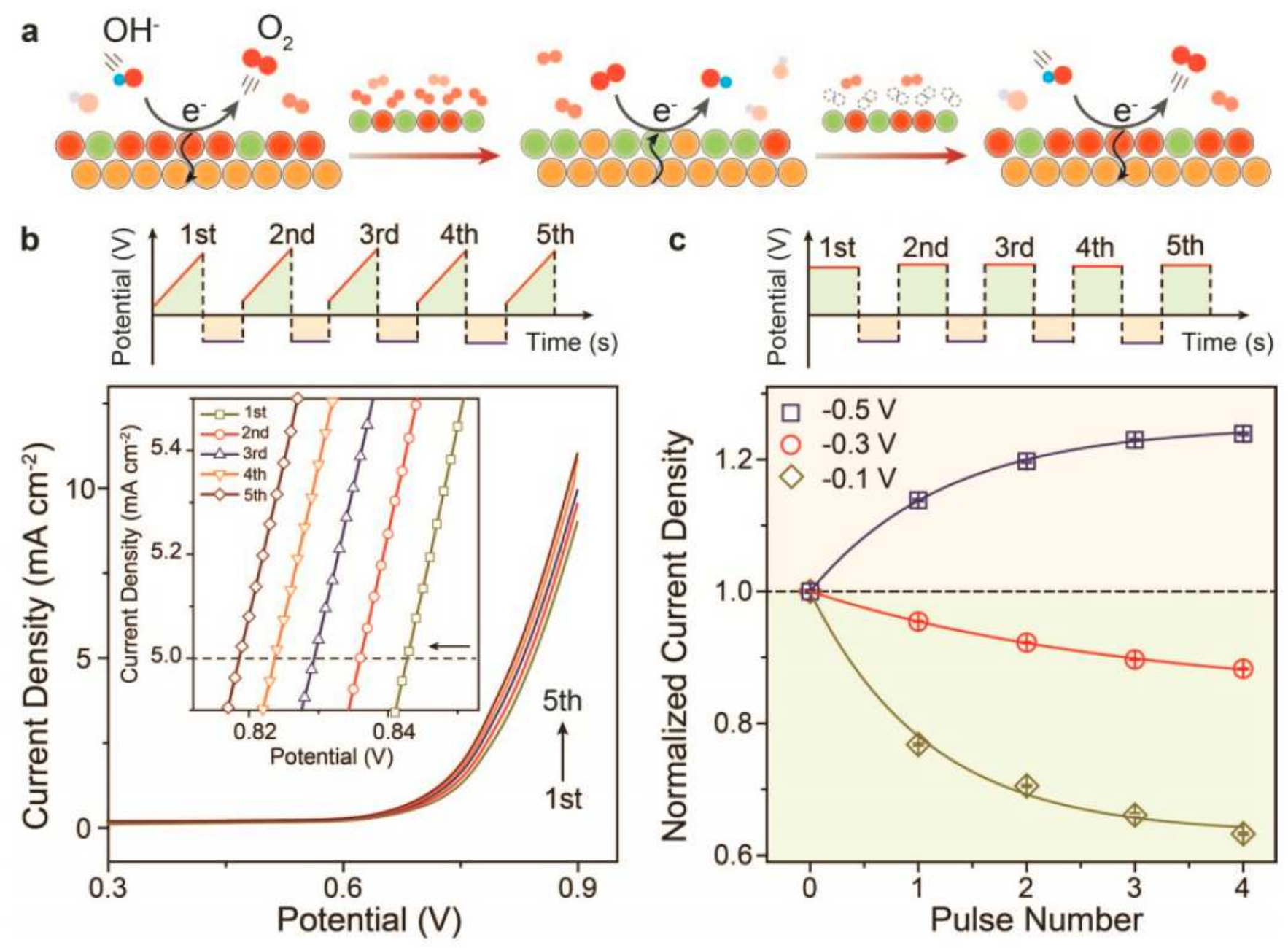

\section{Figure 5}

Programmable potential scan strategy to eliminate accumulated interfacial dissolved oxygen at the EEls. (a) Schematic illustration of reduction potential pulse accelerating interfacial oxygen species remove from the electrode surface. (b) Linear sweep voltammetry curves of Cu electrode combined with reduction potential multi-pulse $(-0.50 \mathrm{~V})$ in $0.10 \mathrm{M} \mathrm{KOH}$ solution at a scan rate of $0.01 \mathrm{~V} \mathrm{~s}-1$. Inset shows cathodic shift of the oxygen evolution potential as consecutive reduction potential pulses $(-0.50 \mathrm{~V})$. (c) Mean chronoamperometry current density at $0.70 \mathrm{~V}$ as a function of reduction potential multi-pulse $(-0.50,-$ 0.30 and $-0.10 \mathrm{~V}$ ) normalized by the value of the original current density (1st scan). Schematic of the programmed potential scan strategy shown in upper panel in (b) and (c), respectively.

\section{Supplementary Files}

This is a list of supplementary files associated with this preprint. Click to download.

- NCSI20201109.docx 